\title{
An Support Vector Regression-Based Data-Driven Leaflet Modeling Approach for Personalized Aortic Valve Prosthesis Development
}

\author{
Jannis Hagenah $^{1}$, Tizian Evers ${ }^{1}$, Michael Scharfschwerdt ${ }^{2}$, Achim Schweikard ${ }^{1}$, Floris Ernst ${ }^{1}$ \\ ${ }^{1}$ Institute for Robotics and Cognitive Systems, University of Lübeck, Lübeck, Germany \\ ${ }^{2}$ Department of Cardiac Surgery, University Hospital Schleswig-Holstein, Lübeck, Germany
}

\begin{abstract}
While the aortic valve geometry is highly patientspecific, state-of-the-art prostheses are not aiming at reproducing this individual geometry. One challenge in manufacturing personalized prostheses is the mapping from the curved $3 D$ shape extracted from imaging modalities to the planar $2 D$ leaflet shape that is cut out of the fabrication material. To address this problem, a database was set up to evaluate valve leaflet shape models. First, $3 D$ ultrasound images of ex-vivo porcine valves were acquired under physiologically realistic pressure to extract geometric key parameters describing the individual geometry. In a second step, the valves' leaflets were cut out, spread on an illuminated plate and photographed in this state. From these images, the leaflet shape was extracted using edge detection. This database allows the derivation of a datadriven leaflet model utilizing machine learning, i.e. nonlinear Support Vector Regression (SVR). Additionally, an existing geometric leaflet shape model was evaluated on the dataset. The data-driven approach provided an acceptable leaflet shape estimation and clearly outperformed the existing model. This presents an important step towards personalized aortic valve prostheses.
\end{abstract}

\section{Introduction}

The geometry of the aortic valve is highly patientspecific [1]. The shape of each of the three leaflets depends on the individual geometry of the aortic root as well as the shape of the other two leaflets. Studies indicate that even small changes in this geometry have a significant influence on the whole blood cycle, up to an increased short-term mortality [2].However, state-of-the-art aortic valve prostheses are hardly capable of reproducing this individual valve shape. While mechanical prosthesis are shaped completely differently, biological aortic valve prostheses are always symmetric, i.e. the three leaflets are equally sized and shaped [3]. A personalization of the prosthesis geometry could improve the patient's outcome. One prob- lem is to estimate the planar shape of the single leaflets. Information about the original valve geometry can only be assessed using medical imaging. In these images, the leaflets appear in a curved three-dimensional shape, while the manufacturing process requires a planar shape description of the leaflets to cut them out of the fabrication material. Accordingly, a model mapping from key features of the curved valve geometry to the planar leaflet shape is required. In combination with a personalized stent, such a model would present a straight-forward approach for personalized valve prosthesis manufaction. The derivation of this model can be performed data-driven using machine learning methods. Like this, systematical errors introduced through handcrafted, geometrical modeling can be reduced. In this work, we present a method to develop and evaluate such a model. It is based on the results of our preliminary study [4].

For this purpose, we set up a sufficient data base and derived a predictive model from it completely data-driven utilizing Support Vector Regression. We examined different key feature descriptors of the 3D aortic valve geometry and evaluated the resulting models on the data set. Additionally, we evaluated an existing geometric leaflet shape model on the data base and compared the results to the data-driven approach.

\section{Material and Methods}

The aim of this work is the derivation of a model mapping from the curved 3D valve geometry to the planar shape of the single leaflets. Hence, a sufficient data base integrates both states of the valve leaflets. Thus, we set up such a data base consisting of 10 ex-vivo porcine aortic roots. At first, volumetric ultrasound images of the aortic root were taken. Then, the single leaflets were cut out and photographed. Thus, the data base consists of corresponding information about each valve's geometry as well as its leaflets' shapes. Based on this data, a mapping was derived utilizing Support Vector Regression. 


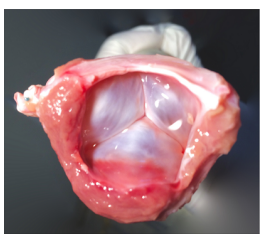

(a)

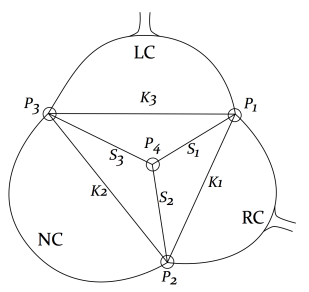

(c)

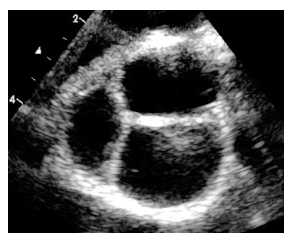

(b)

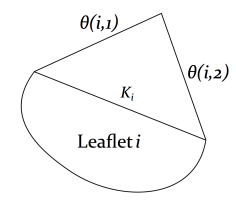

(d)
Figure 1. (a) Porcine aortic valve ex-vivo under pressure, seen from below. (b) Ultrasound image of aortic valve at coaptation plane. (c) Geometric key features of the rightcoronary (RC), left-coronary (LC) and non-coronary (NC) leaflets extracted from the three commissure points $P_{1}, P_{2}$ and $P_{3}$ and the coaptation point $P_{4}$. The features are the commissure distances $K_{1}, K_{2}$ and $K_{3}$ as well as the free leaflet edges $S_{1}, S_{2}$ and $S_{3}$. The image is a projection of the 3D root to the $x-y$-plane. (d) General description of the $i$ th leaflet.

\subsection{Curved Shape Acquisition}

The gold standard for aortic valve imaging is transesophageal echocardiography (TEE). Hence, volumetric ultrasound images of the aortic root under diastolic pressure were taken, i.e. in the closed state, mimicking a TEE examination [5] (see Fig. 2 (a,b)). In these images, four anatomical landmarks were manually identified, being the three commissure points $P_{1}, P_{2}$, and $P_{3}$ as well as the coaptation point $P_{4}$ (see Fig. 2 (c)). Previous studies have shown that this simple geometric description allows for a reasonable representation of the individual geometry [6]. Based on these landmarks, different geometric key features were extracted, namely the commissure distances $K_{1}, K_{2}$ and $K_{3}$ as well as the leaflets' free edge lengths $S_{1}, S_{2}$ and $S_{3}$ (see Fig. 2 (c)).

\subsection{Planar Shape Acquisition}

After volumetric image acquisition, the single leaflets were cut out of the root. They were spread on an illuminated plate and a photograph was taken. The backillumination with blue light increased the contrast through absorption in the prominent collagen fibres. Attention has been paid to the preservation of the natural shape with minimal deformation. The resolution of the photograph was $27.2 \frac{\mathrm{pixel}}{\mathrm{mm}}$. In these images, the leaflet contour line was identified automatically (see Fig. 3). The image was converted to grayscale intensity values and inverted. Then, the leaflet was segmented using thresholding, followed by a closing operation (circular structural element with radius of 30 pixels). Finally, the leaflet contour was extracted using the Canny algorithm for edge detection. For a more general description of the contour line, it was transformed to polar coordinates with its origin in the nodulus arantius, i.e. the point on the leaflet's free edge where it touches both other leaflets (see Fig. 2 (c)).

\subsection{Data-Driven Model Derivation}

The data base described above allows for the derivation of an intelligent system to estimate the mapping of the geometric key features extracted from the ultrasound images to the leaflet contour shape. Thus, the aim is to find a mapping $F_{i}$ for each leaflet $i \in\{1,2,3\}$ with

$$
F_{i}(\phi, x) \rightarrow \rho,
$$

where $\phi$ is the angle of the polar contour coordinate system, $\rho$ is the radius in this system and $x \in$ $\left\{X_{1}(i), X_{2}(i), X_{3}(i), X_{4}(i)\right\}$ is a vector containing a set of individual geometric key features extracted from the ultrasound images. Thus, the leaflet contour line can be estimated for a discretized grid of angles depending on the curved valve shape represented by $x$. The parameter $i$ is the leaflet index and indicates whether the right-coronary $(i=1)$, non-coronary $(i=2)$ or left-coronary $(i=3)$ leaflet shape is predicted. In this study, four different sets of key feature descriptions were examined to analyse the influence of different features on the problem of personalization. The new features $S_{t}$ and $K_{t}$ were defined as

$$
\begin{aligned}
S_{t} & =S_{1}+S_{2}+S_{3} \\
K_{t} & =K_{1}+K_{2}+K_{3} .
\end{aligned}
$$

$S_{t}$ is the sum over all free edge lengths while $K_{t}$ is the sum over the commissure distances. Hence, these new features allow an estimation of the valve's overall size. By division through them, a feature can be scaled to this size approximation. Accordingly, the four examined sets of geometric key features were defined as 


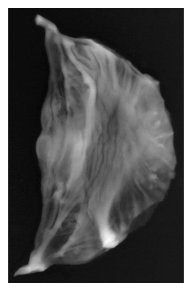

(a)

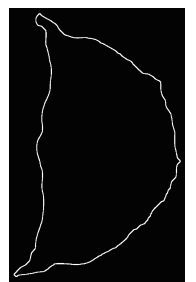

(b)

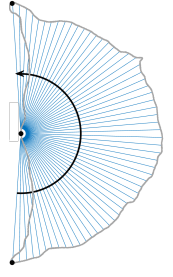

(c)
Figure 2. Planar leaflet shape extraction. (a) Inverted absorption image of leaflet. (b) Extracted contour line using Canny edge detection after segmentation. (c) Contour description in polar coordinates.

$$
\begin{aligned}
& X_{1}(i)=\left\{\frac{\theta(i, 1)}{S_{t}}\right\} \\
& X_{2}(i)=\left\{\frac{\theta(i, 1)}{S_{t}}, \frac{\theta(i, 2)}{S_{t}}\right\} \\
& X_{3}(i)=\left\{\frac{\theta(i, 1)}{S_{t}}, \frac{\theta(i, 2)}{S_{t}}, K_{i}\right\} \\
& X_{4}(i)=\left\{\frac{\theta(i, 1)}{S_{t}}, \frac{\theta(i, 2)}{S_{t}}, \frac{K_{i}}{K_{t}}\right\},
\end{aligned}
$$

where $i$ is the leaflet index, $K_{i}$ is this leaflet's commissure distance and $\theta(i, j), j \in\{1,2\}$, is the $j$ th free edge length of the leaflet $i$ (see Fig. $2(\mathrm{~d})$ ).

The four key feature descriptions differ in their complexity. Due to the relatively small number of samples, low dimensional descriptions where tested. While $X_{1}(i)$ only takes one of the leaflet's free edge lengths into account, $X_{2}(i)$ considers both free edge lengths to ensure a better representation of the leaflets' shearing. By division through $S_{t}$, the key features were scaled according to an estimate of the valve's size. In addition, the commissure distance of the leaflet is included in $X_{3}(i)$ and $X_{4}(i)$ to measure the general span of the leaflet. In this case, the absolute distance $\left(X_{3}(i)\right)$ as well as the distance scaled to the sum of commissure distances $\left(X_{4}(i)\right)$ was examined. The mapping $F_{i}$ was estimated using Support Vector Regression (SVR) [7] with a radial basis function kernel, utilizing the LIBSVM library [8]. To ensure that asymmetric geometries can be estimated, three different models were trained predicting the shape of one specific leaflet, i.e. the left-coronary leaflet, the right-coronary leaflet and the noncoronary leaflet, respectively. The SVR parameters $C$ and $\varepsilon$ as well as the kernel parameter $\gamma$ were optimized using a grid search method $(C \in[0.01,1000], \varepsilon \in[0.01,1], \gamma \in$ $[1,1100]$, discretization: 100 steps, respectively). 10-foldcrossvalidation was performed, i.e. the model was trained on nine valves and evaluated on the last one by predicting its shape and comparing it to the ground truth.

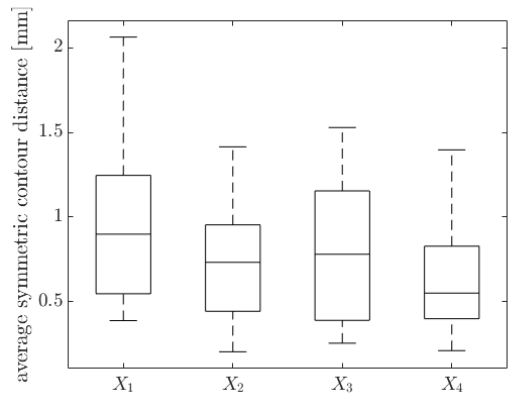

Figure 3. Prediction accuracy of the data-driven model for the four different feature descriptions.

\subsection{Comparison to Existing Model}

For a better interpretation of the model accuracy, the geometric leaflet shape model of Sievers et al. [9] was evaluated to analyse its capability of reproducing personalized leaflet shapes. In this model, while the contour line is modelled by adding three circle sections with different radii [9]. The ratio of these radii was derived empirically. The model creates symmetric leaflets, hence each leaflet is meant to span around the same compartment of the aortic root, which is one third of the root circumference. To reach personalization, the radii were scaled according to the commissure distance $K_{i}$ of the specific leaflet $i, i \in 1,2,3$. This leads to three individually shaped leaflets that span the entire circumference.

\section{Results and Discussion}

The data-driven model was evaluated for the four different key feature descriptions on all ten heart valves for each leaflet, respectively, resulting in 30 predictions per model. Fig. 4 shows the average symmetric contour distance for each examined feature space. The scaling of the features to measures of the valve's size $\left(X_{2}, X_{4}\right)$ clearly improves the prediction accuracy. A combined geometry description using the leaflets' free edge lengths and the commissure distance in this scaled representation $\left(X_{4}\right)$ provided the smallest errors. Therefore, the following predictions were based on this feature space. Additionally, the data-driven model with the optimal feature space as well as the model of Sievers et al. were evaluated on the data set. Table 1 shows the prediction accuracy of both models for all three leaflets as well as the mean prediction accuracy. While the error of Sievers et al.'s approach lies above $2 \mathrm{~mm}$, the data-driven model can predict each leaflet's geometry with an error of less than $1 \mathrm{~mm}$.

The results show that the geomtric model of Sievers et al. is not capable of reproducing patient-specific leaflet 
shapes. This indicates that current models for heart valve prosthesis are still far from optimal. In contrast, the datadriven model provides good approximations and clearly outperforms the existing model. Hence, it could be shown that estimating the individual leaflet shape based on ultrasound image data is possible. Additionally, a feasible representation of the valve's shape based on four anatomical landmarks was found and it could be shown that personalized estimation of the leaflet shapes can be performed even based on this simple geometric description.

In both approaches, the non-coronary leaflet can be modelled with the highest accuracy. This could be due to the fact that this leaflet is more likely to be symmetric, while the right- and left-coronary leaflets are more likely to appear sheared, i.e. the nodulus does not lie in the middle of the leaflet's free edge.

Due to several manual interaction steps, it is possible that the data set is biased. The first possible reason could be inaccuracies in the manual landmark identification in the ultrasound volume images, though the results indicate that the manual geometry description worked accurately. A second source of error in the data set is due to the manual spreading of the single leaflets on the illuminated plate. Positioning errors will affect the assessed leaflet contour shape. Hence, the sample size should be increased to statistically minimize this error. Additionally, this bigger data set could increase the accuracy of the learning algorithm.

Aiming for the manufacturing of personalized aortic valve prosthesis, modelling the planar leaflet is just one step. Additionally, a personalized model of the stent has to be developed. Another interesting scientific question is whether the obtained individual geometry of the valve is pathologically changed. In this case, a direct copy of the valve geometry would not increase the patient's outcome. One possibility to overcome this problem could be an additional estimation step predicting the healthy geometry based on the obtained one. A similar approach was already tested in a comparable cardiovascular problem [6].

To the best of the author's knowledge, this work presents the first method to assess the planar shape of the aortic valve leaflets based on ultrasound imaging. Based on an expermentally collected data set, an existing method to

Table 1. Prediction accuracy of the two evaluated models for each leaflet type. All values given as the average symmetric contour distance in $\mathrm{mm}$.

\begin{tabular}{lcc}
\hline & Sievers et al. & Data-driven Model \\
\hline Left-coronary & $2.77 \pm 0.84$ & $0.69 \pm 0.23$ \\
Right-coronary & $2.96 \pm 0.73$ & $0.64 \pm 0.31$ \\
Non-coronary & $2.65 \pm 0.70$ & $0.41 \pm 0.48$ \\
\hline Mean & $2.21 \pm 0.56$ & $0.61 \pm 0.33$ \\
\hline
\end{tabular}

compute valve prostheses shapes was evaluated. Furthermore, the data base allows the derivation of a completely data-driven model using machine learning. It was shown that this model clearly outperformed the existing model of Sievers et al. and that acceptable prediction accuracies were reached. The developed model makes the fabrication of personalized aortic valve prostheses possible. To this end, TEE images of the patient can be acquired and, based on this information, the three leaflet shapes can be predicted using the model. In combination with a personalized stent model, a prosthesis can be manufactured that mimics the original geometry of the patient's own aortic valve. The study shows that machine learning could be an important step towards personalized cardiovascular prostheses.

\section{References}

[1] Michel R Labrosse, Carsten J Beller, Francis Robicsek, and Mano J Thubrikar. Geometric modeling of functional trileaflet aortic valves: development and clinical applications. Journal of Biomechanics, 39(14):2665-2672, 2006.

[2] Thomas Walther. Patient prosthesis mismatch affects shortand long-term outcomes after aortic valve replacement. $E u$ ropean Journal of Cardio-Thoracic Surgery, 30(1):15-19, 2006.

[3] Philippe Pibarot and Jean G Dumesnil. Prosthetic heart valves. Circulation, 119(7):1034-1048, 2009.

[4] Jannis Hagenah, Tizian Evers, Michael Scharfschwerdt, and Achim Schweikard. Data-driven leaflet modeling for personalized aortic valve prostheses development. Biomedical Engineering/Biomedizinische Technik, 62(s1):s403, 2017.

[5] J Hagenah, M Scharfschwerdt, B Stender, S Ott, R Friedl, HH Sievers, and A Schlaefer. A setup for ultrasound based assessment of the aortic root geometry. Biomedical Engineering/Biomedizinische Technik (2013), 2013.

[6] Jannis Hagenah, Erik Werrmann, Michael Scharfschwerdt, Floris Ernst, and Christoph Metzner. Prediction of individual prosthesis size for valve-sparing aortic root reconstruction based on geometric features. In IEEE 38th Annual International Conference of the Engineering in Medicine and Biology Society (EMBC), 2016, pages 3273-3276. IEEE, 2016.

[7] Alex J Smola and Bernhard Schölkopf. A tutorial on support vector regression. Statistics and Computing, 14(3):199-222, 2004.

[8] Chih-Chung Chang and Chih-Jen Lin. Libsvm: a library for support vector machines. ACM Transactions on Intelligent Systems and Technology (TIST), 2(3):27, 2011.

[9] H.H. Sievers, M. Scharfschwerdt, and A. Hof. Biologische herzklappenprothese. Patent DE102014223522, Germany (2016), 2016.

Address for correspondence:

Jannis Hagenah, hagenah@ rob.uni-luebeck.de

Inst. for Robotics and Cognitive Systems, University of Lübeck 